tion, indicating institutional affiliation and position, and present areas of research and publications related to the topic of the conference ( 400 words). Contributors will be notified about the acceptance of their proposal by mid-May, 2007.

For further information like topics for the proposals of papers please contact Prof. Dr. Max Haller, Institut für Soziologie, Karl FranzensUniversität Graz, Universitätsstraße 15, A-8010 Graz/Austria, Tel. (++43-316) 380 3541;

Fax (--43-316) 380 9515,

E-Mail:max.haller@uni-graz.at.

\title{
Gutachterinnen und Gutachter der KZfSS 2005/2006
}

An der Begutachtung von Manuskripten, die der Zeitschrift im Zeitraum zwischen dem 1. Juli 2005 und dem 30. Juni 2006 eingereicht wurden, haben die folgenden Kolleginnen und Kollegen mitgewirkt. Wir danken ihnen sehr herzlich für die Beteiligung an der Entstehung der Zeitschrift.

\author{
Hans-Jürgen Andreß (Köln) \\ Hans-Peter Blossfeld (Bamberg) \\ Klaus Boers (Münster) \\ Heinz Bude (Kassel) \\ Günter Burkart (Lüneburg) \\ Nicole Burzan (Hagen) \\ Wolfgang van den Daele (Berlin) \\ Klaus Dörre (Jena) \\ Günter Endruweit (Kiel) \\ Andreas Flache (Groningen) \\ Bruno S. Frey (Zürich) \\ Hans-Joachim Gergs (Nürnberg) \\ Peter H. Hartmann (Düsseldorf) \\ Jürgen H.P. Hoffmeyer-Zlotnik (Mannheim) \\ Alois Hahn (Trier) \\ Steffen Hillmert (Bamberg) \\ Stefan Hornbostel (Bonn) \\ Johannes Huinink (Bremen) \\ Matthias Junge (Rostock) \\ Frank Kalter (Leipzig) \\ Udo Kelle (Vechta) \\ Thomas Klein (Heidelberg) \\ Johannes Kopp (Chemnitz) \\ Cornelia Kristen (Leipzig) \\ Steffen-M. Kühnel (Göttingen)
}

\author{
Henning Lohmann (Köln) \\ Martina Löw (Darmstadt) \\ Hans Mathias Kepplinger (Mainz) \\ Kurt Mübler (Leipzig) \\ Günter Naegeler (Aachen) \\ Bernhard Nauck (Chemnitz) \\ Alexandra Nonnenmacher (Köln) \\ Dietrich Oberwittler (Freiburg i.Br.) \\ Sven Papcke (Greven) \\ Michaela Pfadenhauer (Dortmund) \\ Astrid Podsiadlowski (München) \\ Manuela Pötschke (Bremen) \\ Uwe Schimank (Hagen) \\ Volker H. Schmidt (Mannheim) \\ Rainer Schnell (Konstanz) \\ Dirk Tänzler (Konstanz) \\ Heike Trappe (Berlin) \\ Gisela Trommsdorff (Konstanz) \\ Arne Uhlendorff (Berlin) \\ Franz Urban Pappi (Mannheim) \\ Georg Vobruba (Leipzig) \\ Erich Weede (Königswinter) \\ Claus Wendt (Bremen) \\ Michael Windzio (Hannover) \\ Stefanie Würtz (Bielefeld)
}

